\title{
Instrumentos psicológicos: estudo comparativo entre estudantes e profissionais cognitivo-comportamentais
}

\author{
Psychological instruments: comparative study between \\ cognitive-behavioral students and professionals
}

\author{
Katya Luciane de OLIVEIRA ${ }^{1,2,4}$ \\ Ana Paula Porto NORONHA ${ }^{3}$ \\ Marilda Aparecida DANTAS ${ }^{4}$
}

\begin{abstract}
Resumo
Esta pesquisa analisou se há diferença entre os instrumentos psicológicos mais conhecidos e utilizados por estudantes e profissionais cognitivo-comportamentais. Participaram 40 estudantes, do último ano do curso de Psicologia e 35 psicólogos. Foi utilizado instrumento composto de duas partes, a primeira contemplou questões que visavam à caracterização dos sujeitos quanto à formação, atuação profissional, estratégias e instrumentos utilizados na avaliação. A segunda parte constava de uma relação contendo 152 instrumentos de avaliação psicológica, na qual os sujeitos tinham que assinalar os conhecidos e os utilizados. Os resultados evidenciaram que tanto estudantes quanto profissionais utilizam com maior freqüência em sua avaliação as entrevistas. Os instrumentos mais conhecidos pelos sujeitos foram o Zulliger, a Escala de Maturidade Mental Columbia, o Teste de Apercepção Temática e o WISC-III. Os mais utilizados foram o Bender Infantil, o Desenho da Figura Humana e o Teste Wartegg. Sugere-se que novos estudos sejam realizados com o intuito de promover avanços na preparação do psicólogo brasileiro.
\end{abstract}

Palavras-chave: avaliação comportamental; avaliação psicológica; testes psicológicos.

\begin{abstract}
This research analyzed if there was any difference between the well-known psychological instruments and the ones used by cognitive-behavioral students and professionals. 40 students and 35 psychologists participated in this study. This study material was composed by two parts. The first one comprised by questions about graduation, professional activity, strategy and instruments used in the assessment. The second part constituted a set of psychological assessment instruments, and the participants task was to assign if they were known or used. Results indicated the most of students and professionals uses the interview in the assessment. The most well-known instruments were Zulliger, Escala de Maturidade Mental Columbia, Teste de Apercepção Temática and WISC-III. The most used instruments were Bender Infantil, Desenho da Figura Humana and Wartegg Test. Others studies should be made to promote the psychologists' formation development.

Key words: behavioral assessment; psychological assessment; psychologicaltests.

\section{$\operatorname{rrv}$}

' Doutoranda em Psicologia do Desenvolvimento Humano e Educação, Faculdade de Educação, Universidade Estadual de Campinas. Campinas, SP, Brasil.

2 Professora, Curso de Psicologia, Universidade São Francisco. Rua Alexandre Rodrigues Barbosa, 45, Itatiba, SP, Brasil. Correspondência para/Correspondence to:K.L.OLIVEIRA. E-mail:<katya_lincoln@ig.com.br>.

3 Professora Doutora, Curso de Psicologia e do Programa de Pós-Graduação Stricto Sensu em Psicologia, Universidade São Francisco. Itatiba, SP, Brasil.

4 Professora, Curso de Psicologia, Universidade de Alfenas. Alfenas, MG, Brasil.
\end{abstract}


No Brasil, autores têm se dedicado ao desenvolvimento da área de avaliação psicológica. Mesmo que com contribuições isoladas e com objetivos distintos - como oferecer manuais atualizados com os fundamentos técnicos dos instrumentos de avaliação, realizar pesquisas e incentivar a construção de novos materiais -, tais instrumentos tornaram-se o foco de diversos estudos (Pasquali, 1999, 2001).

Ainda em âmbito nacional, o Conselho Federal de Psicologia (CFP) promulgou resoluções (Conselho Federal de Psicologia, 2001, 2003) a fim de oferecer definições sobre o uso, a elaboração e a comercialização dos testes. Embora inicialmente a decisão tenha sido polêmica, considerando o impacto que ocasionou na comunidade científica e entre leigos, acredita-se que o processo promoverá a construção de instrumentos com mais qualidade do ponto de vista psicométrico.

Dentre os elementos definidos pelas resoluções como imprescindíveis quando da construção de testes encontram-se o desenvolvimento de estudos com amostras nacionais e os estudos de evidência de validade e de precisão. Embora os aspectos apontados pareçam elementares, pesquisas indicam que grande parte dos instrumentos comercializados no Brasil ainda não apresenta condições mínimas de comercialização (Noronha, 2003). Poucos são os instrumentos que se encontram com parecer favorável do CFP para utilização. Esse fato ocorre devido à falta de parâmetros psicométricos na maior parte dos instrumentos avaliados (Conselho Federal de Psicologia, 2005), diferente do que ocorre em países mais desenvolvidos, onde é intenso o foco nos atributos psicométricos de um teste.

Nesse sentido, alguns estudos já evidenciaram a necessária atenção que deve ser dispensada aos instrumentos de medida. Andriola (1995) destaca que os testes psicológicos como instrumentos de avaliação merecem mais esmero por parte dos profissionais responsáveis pela utilização, principalmente pelo seu uso freqüentemente indiscriminado. A ênfase deve ser dirigida ao rigor da qualidade científica, estudando os parâmetros psicométricos e atualizando os conteúdos dos instrumentos a fim de contribuir com a valorização da avaliação e do profissional que a realizou (Noronha, 2003; Pasquali, 2001; Wechsler \& Guzzo, 1999).
Não parece ser novidade que os testes divulgam e favorecem o reconhecimento social da profissão e que a má aplicação tende a gerar dificuldades no atendimento desses objetivos, considerando que o início da testagem de alguma forma se confunde com os primórdios da Psicologia científica.

No que se refere às questões éticas e deontológicas da avaliação (considerando-a como um processo de aquisição de informações, que faz uso de vários métodos, técnicas e instrumentos, tendo como objetivo principal a compreensão do indivíduo), Simões (1995) observa que a ética é distinguida da deontologia, sendo a primeira relativa à ordem de questionamento sobre valores e a segunda à moral profissional. Baseado nessa concepção, o autor determina que compete ao psicólogo a responsabilidade da escolha e aplicação de instrumentos com valor reconhecido. Nessa direção, também se faz necessário que tal instrumento psicológico comungue com o referencial teórico do psicólogo; não há como fazer uso de um recurso cujo fundamento teórico seja dissonante daquele que sua abordagem de atuação preconiza.

Quanto à escolha dos instrumentos mais adequados para as diferentes situações profissionais nas quais os psicólogos se deparam ao longo de suas atividades, deve-se evidenciar a eleição de métodos e de instrumentos que tenham pesquisas relacionadas aos parâmetros técnicos científicos (precisão, validade e normas). Tal asserção é corroborada pelos estudos de Oliveira, Noronha, Beraldo e Santarem (2003) e Oliveira, Noronha, Dantas e Santarem (2005), no sentido de que é imprescindível que haja coerência entre a abordagem teórica que norteia a prática profissional do psicólogo e os construtos dos instrumentos utilizados, de modo que a leitura de homem adotada esteja em consonância com a que o instrumento preconiza.

No que tange à avaliação comportamental, vale ressaltar que há diferenças em relação a outras abordagens, principalmente no que concerne ao conjunto de princípios e elementos conceituais. Alguns dos princípios da avaliação comportamental são comuns a qualquer sistema de avaliação, outros, porém, são específicos e refletem os princípios comportamentais de base, muitos dos quais são derivados da análise experimental do comportamento (Matos, 1999; Santarem, 2001). 
A avaliação comportamental pode ser considerada um termo genérico ou um paradigma da avaliação psicológica que abriga diferentes abordagens ou subparadigmas, como, por exemplo, análise funcional e cognitivo-comportamental. O termo comportamento implica a relação do organismo com o ambiente num processo contínuo e dinâmico (Delitti, 2001; Meyer, 2001; Rangé \& Guilhardi, 1998; Torós, 1997).

Meyer (2001) observa que o psicólogo comportamental pode recorrer aos testes e inventários em uma avaliação. Todavia, de acordo com Piotrowski e Lubin (1990) e Haynes e O'Brien (2000), tais instrumentos devem ser entendidos como recursos auxiliares ao processo de coleta de dados, já que são construídos tendo como base a teoria psicométrica.

Keepe, Kopel e Gordon (1980) esclarecem que os testes e técnicas projetivas não devem ser utilizados em nenhuma instância pelos terapeutas comportamentais. Estudos destinados a avaliar a coerência teórica e filosófica entre a abordagem adotada e os instrumentos utilizados na avaliação são quase inexistentes. Cabe questionar, portanto, se tal coerência vem sendo respeitada.

O estudo de Oliveira, Noronha, Dantas e Santarem (2005) desenvolvido com psicólogos comportamentais evidenciou que os instrumentos mais utilizados são os de base analítica e ainda são os mais ensinados na universidade. Fato semelhante foi observado por Watkins, Campbell e McGregor quando em 1990 realizaram um estudo sobre o assunto. Na verdade, a comparação entre os mais usados e aprendidos só foi possível ao analisar os estudos de Alves, Alchieri e Marques (2001), cujo objetivo foi mapear o ensino da avaliação psicológica no Brasil, inclusive no que diz respeito aos instrumentos preferencialmente ministrados nas universidades.

Observando especialmente a formação profissional, há que se ressaltar a situação do ensino de testes psicológicos brasileiros. Noronha (2003) afirma que é preciso repensar a formação do profissional de Psicologia de modo que o psicólogo tenha uma bagagem teórica mais consistente e faça um uso ético e consciente dos instrumentos.

A formação profissional tem sido tema freqüente em discussões promovidas por órgãos e associações de classe. Recentemente, o Conselho Regional de Psicologia - 6a Região, em consonância com outras entidades, organizou um encontro cujo foco era o ensino da avaliação psicológica (Conselho Regional de Psicologia, 2004). Embora eventos nem sempre possam oferecer soluções imediatas para as questões emergentes, vale ressaltar que tais discussões fomentam reflexões e tendem a gerar pesquisas.

A formação em avaliação psicológica no Brasil é ainda incipiente e o reflexo disso recai na prática profissional. Muito se tem discutido sobre a melhor forma de ensinar instrumentos específicos ou a avaliação mais amplamente definida. As tendências são as mais diversas possíveis, variando desde a construção de um currículo mínimo para a área (Jacquemin, 1995), até a inserção do ensino da avaliação do desenvolvimento em outras disciplinas afins (Kroeff, 1988), como, por exemplo, na disciplina Psicologia do Desenvolvimento, ou ainda ensinar a construção dos testes (Pasquali, 2001).

As discussões, embora ainda não tenham gerado encaminhamentos definitivos, são extremamente positivas, pois possibilitam outras análises acerca das práticas ora existentes. Alchieri e Bandeira (2002) enfatizam que em grande parte dos cursos de Psicologia no Brasil, a opção de ensino de avaliação ainda se dá por meio de informações sobre a aplicação, a avaliação e a interpretação de instrumentos. Pouco se fala em construção de testes ou em parâmetros psicométricos.

Da forma como o conteúdo de avaliação vem sendo passado não tem sido possível desenvolver análises mais críticas a respeito dos instrumentos, do uso e, sobretudo, das limitações. O resultado desse processo equivocado é a formação de profissionais com conhecimento bastante restrito, dominando apenas a aplicação e a correção de poucos instrumentos. Guzzo (2001) aponta que aprender técnicas do exame psicológico de forma isolada e pontual não assegura as competências necessárias, como, por exemplo, para, dentre outras tarefas, chegar a conclusões ou elaborar laudos. Sob essa perspectiva da formação profissional, o objetivo desse estudo foi analisar se há diferença entre os instrumentos psicológicos mais conhecidos e utilizados por estudantes e profissionais cognitivo-comportamentais. 


\section{Método}

\section{Participantes}

Participaram 75 sujeitos, sendo 40 (53,3\%) estudantes de Psicologia que haviam optado pelo estágio em clínica comportamental e 35 (46,7\%) psicólogos, cuja abordagem de atuação era a cognitivo-comportamental. A média de idade foi de 28 anos e 8 meses ( $D P=9,3)$, com um mínimo de 21 anos e o máximo de 60. O sexo feminino representou $88 \%(n=66)$ dos sujeitos e o masculino 12\% ( $n=9)$.

\section{Instrumentos}

Utilizou-se um instrumento composto de duas partes. A primeira continha questões que visavam à caracterização dos sujeitos quanto à formação, abordagem terapêutica, atividades profissionais e instrumentos utilizados na avaliação.

A segunda parte constituiu-se de uma relação contendo instrumentos de avaliação psicológica específicos. Na relação estavam presentes 152 instrumentos das seguintes editoras: Cepa, Vetor, Casa do Psicólogo, Edites, Cetepp, Artes Médicas, Editorial Psy, Mestre Jou, Melhoramentos, Entreletras, Gráfica MNJ Ltda e Martins Fontes, além de dez testes estrangeiros e dois testes de editoras não localizadas. Os sujeitos tiveram duas possibilidades de resposta para cada instrumento listado, a saber: 'C' para os instrumentos conhecidos e'U'para os utilizados. Caso o instrumento não fosse utilizado ou conhecido, o sujeito não deveria fazer nenhuma anotação.

\section{Procedimentos}

A coleta de dados ocorreu em universidades dos estados de Minas Gerais e de São Paulo, no VI Congresso Brasileiro de Psicologia Escolar e Educacional e no I Congresso Nacional de Psicologia. Em todas as ocasiões a aplicação foi individual, e o instrumento auto-aplicável. Aqueles que optaram por participar da pesquisa respondiam ao instrumento, entregando-o, em seguida, às pesquisadoras. Destaca-se que esta pesquisa foi aprovada pelo comitê de ética em pesquisa da instituição à qual está vinculada.

\section{Resultados e Discussão}

Após a coleta, os dados foram organizados em planilhas e submetidos à estatística descritiva, de acordo com os objetivos do estudo. A análise foi realizada a partir da freqüência das respostas e das respectivas porcentagens.

Quanto à atuação profissional dos estudantes, $57,5 \%(n=23)$ exerciam alguma atividade profissional, não tendo respondido à questão 42,5\% ( $n=17)$. No caso dos profissionais uma parte tinha somente graduação em Psicologia (31,4\%; $n=11)$, os demais $(68,6 \%$; $n=24)$ tinham algum curso de especialização ou mestrado e doutorado. A atuação em clínicas particulares foi apontada por 48,6\% ( $n=17)$ e 51,4\% $(n=18)$ indicaram que atuam no contexto universitário como docentes e não têm atuação em clínica.

Embora seja um estudo com estudantes e profissionais que atuam na área cognitivo-comportamental, salienta-se que este trabalho contou com uma amostra coletada em universidades e principalmente em congressos não específicos dessa área. Desse modo, o viés da amostra pode ser evidenciado pelo elevado percentual de docentes, considerando que tais eventos são bastante freqüentados por professores e pesquisadores.

O modelo comportamental cognitivo foi o mais citado pela maioria dos estudantes (85\%; n=34) e dos profissionais $(68,6 \% ; n=24)$. Sob esse aspecto Rangé e Guilhard (1998) apontam que a terapia cognitivo-comportamental é uma prática crescente com uma aceitação progressiva.

A Tabela 1 apresenta as considerações sobre a utilização de estratégias e instrumentos gerais na avaliação realizada pelos estudantes e profissionais. Nessa questão, os participantes tinham que assinalar com que freqüência recorria ao uso das estratégias e instrumentos em sua avaliação.

Evidencia-se que tanto estudantes (50\%; $n=20)$ quanto profissionais (88,6\%; n=31) utilizavam com maior freqüência em sua avaliação as entrevistas, seguidas pela técnica de observação realizada por observadores externos no ambiente natural $(42,5 \%, n=17 ; 54,3 \%, n=19$, respectivamente). Em terceiro lugar como mais utilizada para os estudantes aparece a observação qualitativa 
Tabela 1. Distribuição das freqüências e porcentagens de utilização de estratégias e instrumentos gerais de avaliação ( $n=75)$.

\begin{tabular}{|c|c|c|c|c|c|c|c|c|}
\hline \multirow{2}{*}{ Estratégias e Instrumentos } & \multicolumn{2}{|c|}{ Sempre } & \multicolumn{2}{|c|}{ Ocasionalmente } & \multicolumn{2}{|c|}{ Nunca } & \multicolumn{2}{|c|}{$\begin{array}{c}\text { Não } \\
\text { respondentes }\end{array}$} \\
\hline & $F$ & $\%$ & $F$ & $\%$ & $F$ & $\%$ & $F$ & $\%$ \\
\hline Entrevistas & 51 & 68,0 & 14 & 18,7 & 0 & 0 & 10 & 13,3 \\
\hline Escalas de classificação do comportamento & 16 & 21,3 & 29 & 38,7 & 17 & 22,7 & 13 & 17,3 \\
\hline Inventários de personalidade & 14 & 18,7 & 32 & 42,6 & 14 & 18,7 & 15 & 20,0 \\
\hline Observação participante & 21 & 28,0 & 13 & 17,3 & 21 & 28,0 & 20 & 26,7 \\
\hline $\begin{array}{l}\text { Observação por meio de instrumentos (ex.gravação de } \\
\text { discussão família) }\end{array}$ & 8 & 10,6 & 14 & 18,7 & 33 & 44,0 & 20 & 26,7 \\
\hline $\begin{array}{l}\text { Observação por observadores externos no ambiente } \\
\text { controlado }\end{array}$ & 14 & 18,7 & 22 & 29,3 & 21 & 28,0 & 18 & 24,0 \\
\hline Observação por observadores externos no ambiente natural & 36 & 48,0 & 14 & 18,7 & 10 & 13,3 & 15 & 20,0 \\
\hline $\begin{array}{l}\text { Observação qualitativa durante entrevistas ou sessões } \\
\text { terapêuticas }\end{array}$ & 21 & 28,0 & 16 & 21,2 & 19 & 25,4 & 19 & 25,4 \\
\hline Questionários & 22 & 29,3 & 36 & 48,0 & 7 & 9,4 & 10 & 13,3 \\
\hline Registros de auto-observação do cliente & 37 & 49,4 & 16 & 21,3 & 19 & 25,3 & 3 & 4,0 \\
\hline Técnicas projetivas gráficas & 10 & 13,3 & 21 & 28,0 & 23 & 30,7 & 21 & 28,0 \\
\hline Testes psicológicos & 15 & 20,0 & 28 & 37,3 & 15 & 20,0 & 17 & 22,7 \\
\hline
\end{tabular}

durante entrevistas ou sessões terapêuticas (27,5\%; $\mathrm{n}=11$ ), e para os profissionais, o mais utilizado foi o questionário (42,8\%; $n=15)$.

Nesse sentido, observa-se que na abordagem comportamental o psicólogo busca outros recursos para testar suas hipóteses acerca do fenômeno observado (Matos, 1999). Desse modo, os dados mostraram que tanto estudantes como profissionais recorrem a alguns recursos e técnicas (entrevistas, observação por observadores externos no ambiente natural, observação qualitativa durante entrevistas ou sessões terapêuticas e questionários) para submeterem suas hipóteses à verificação.

Visando identificar quais instrumentos psicológicos específicos eram conhecidos e utilizados pelos sujeitos, foi apresentada uma relação de instrumentos psicológicos específicos de avaliação, na qual estudantes e profissionais tinham que assinalar ' $C^{\prime}$ (se conhecia o instrumento) e ' $U$ ' (se o instrumento era utilizado). Os dados descritos na Tabela 2 apresentam os resultados de instrumentos que são conhecidos por pelo menos dez participantes, destacando que os demais instrumentos ou tiveram pontuações menores do que a citada ou não foram escolhidos.

Os instrumentos mais conhecidos pelos participantes foram em primeiro lugar o Zulliger $(42,5 \%, n=17$ estudantes e $57,5 \%, n=23$ profissionais), em segundo lugar a Escala de Maturidade Mental Columbia e o Teste de Apercepção Temática (51,3\%, n=20 estudantes e $48,7 \%, n=19$ profissionais, respectivamente) e em terceiro lugar a Escala de Inteligência Wechsler para Crianças (WISC-III) (60,5\%, n=23 estudantes e 39,5\%, n=15 profissionais). O teste Qui-quadrado mostrou que a distribuição das citações sobre os instrumentos psicológicos mais conhecidos pela amostra como um todo era eqüitativa. Os dados não evidenciaram diferença significativa entre as citações.

O instrumento mais citado pelos estudantes como conhecido foi o WISC-III. Tal dado pode ser justificado em razão da padronização do instrumento no Brasil ter sido feita em 2002, realizada por Vera Lúcia Marques de Figueiredo. Nessa direção, é provável que o maior conhecimento de estudantes se justifique em razão da aprendizagem mais recente nos cursos de Psicologia. Vale destacar que a educação continuada e o aperfeiçoamento profissional nem sempre estão presentes na prática psicológica.

No caso dos profissionais o instrumento mais conhecido foi o Zulliger. Esse dado talvez esteja relacionado à avaliação clínica tradicional que muitas vezes recorre ao Teste de Rorschach, instrumento bastante utilizado em diversos países, ou ao próprio Zulliger, que pode ser considerado uma versão reduzida do Rorschach com aspectos semelhantes entre si. 
Tabela 2. Distribuição das freqüências e porcentagens referentes aos instrumentos psicológicos mais conhecidos por estudantes e psicólogos comportamentais $(n=75)$.

\begin{tabular}{|c|c|c|}
\hline Testes mais conhecidos & $F$ & $\%$ \\
\hline Teste Zulliger & 40 & 53,3 \\
\hline Teste de Apercepção Temática (TAT) & 39 & 52,0 \\
\hline Escala de Maturidade Mental Columbia & 39 & 52,0 \\
\hline Escala de Inteligência Wechsler 3a edição & 38 & 50,7 \\
\hline Desenho da Figura Humana & 36 & 48,0 \\
\hline Escala de Inteligência Wechsler Crianças $1^{a}$ edição & 36 & 48,0 \\
\hline Dezesseis Fatores de Personalidade & 36 & 48,0 \\
\hline Teste de Rorschach & 34 & 45,3 \\
\hline Teste de Apercepção Infantil CAT-A & 34 & 45,3 \\
\hline Teste de Apercepção Infantil CAT-H & 33 & 44,0 \\
\hline Teste de Inteligência Não Verbal G36 & 31 & 41,3 \\
\hline Teste das Fábulas & 31 & 41,3 \\
\hline Bateria CEPA & 31 & 41,3 \\
\hline Escala de Stress Adulto & 30 & 40,0 \\
\hline Bender Infantil & 30 & 40,0 \\
\hline TesteWartegg & 28 & 37,3 \\
\hline Inventory Multiphasic Minnesota Personality (MMPI) & 28 & 37,3 \\
\hline Supl. Teste Apercepção Infantil CAT-S & 26 & 34,7 \\
\hline Matrizes Progressivas - escala geral & 26 & 34,7 \\
\hline Teste de Inteligência Não Verbal G38 & 25 & 33,3 \\
\hline Matrizes Progressivas - escala avançada & 25 & 33,3 \\
\hline Atenção Concent & 25 & 33,3 \\
\hline Coloridas & 24 & 32,0 \\
\hline a crianças & 23 & 30,7 \\
\hline s Angelini e Thustone & 23 & 30,7 \\
\hline de Interesses Profissionais & 22 & 29,3 \\
\hline s D-48 & 22 & 29,3 \\
\hline imides Coloridas & 21 & 28,0 \\
\hline Inventário Fatorial de Personalidade & 21 & 28,0 \\
\hline Bateria de Testes de Aptidão (BTAG) & 20 & 26,7 \\
\hline Cubos de Kohs & 19 & 25,3 \\
\hline Teste Psicodiagnóstico Miocinético (PMK) & 18 & 24,0 \\
\hline Teste de Aptidões Específicas (DAT) & 18 & 24,0 \\
\hline BPR-5 & 18 & 24,0 \\
\hline Teste das Pirâmides das Cores 24M Pfister & 17 & 22,7 \\
\hline sses Profissionais & 17 & 22,7 \\
\hline insiedade (IDATE) & 17 & 22,7 \\
\hline Palográfico & 16 & 21,3 \\
\hline nventário Interesses Profissionais & 16 & 21,3 \\
\hline ilidades Sociais & 16 & 21,3 \\
\hline letropolitano & 14 & 18,7 \\
\hline $\begin{array}{l}\text { Escala de Transtorno de Déficit de } \\
\text { Atenção/Hiperatividade }\end{array}$ & 14 & 18,7 \\
\hline Escala de Stress Infantil (ESI) & 14 & 18,7 \\
\hline Questionário Vocacional de Interesses & 13 & 17,3 \\
\hline Inventário de Atitudes de Trabalho (IAT) & 13 & 17,3 \\
\hline INV & 13 & 17,3 \\
\hline Teste Não & 12 & 16,0 \\
\hline Programação Hábitos e Desempenho (PHD) & 12 & 16,0 \\
\hline Como Chefiar? & 12 & 16,0 \\
\hline Teste Raven Operações Lógicas (RTLO) & 11 & 14,7 \\
\hline Figuras Complexas de Rey & 11 & 14,7 \\
\hline Diagnóstico Organizacional & 11 & 14,7 \\
\hline Teste Coletivo Inteligência Adultos (CIA) & 10 & 13,3 \\
\hline Quati Questionário Avaliação Tipológica & 10 & 13,3 \\
\hline Lista de Problemas Pessoais Adultos & 10 & 13,3 \\
\hline Inventário de Ansiedade IDATE-C & 10 & 13,3 \\
\hline Escala de Maturidade Escolha profissional & 10 & 13,3 \\
\hline Atenção Concentrada 15 & 10 & 13,3 \\
\hline
\end{tabular}

Na Tabela 3 foram elencados os resultados dos instrumentos utilizados por pelo menos oito sujeitos. Os demais instrumentos ou tiveram pontuações menores dos que as citadas ou não foram escolhidos.

No caso dos instrumentos mais utilizados pelos participantes observou-se que em primeiro lugar apareceu o Bender Infantil (48,5\%, n=17 estudantes e $51,5 \%, n=18$ profissionais), em segundo o Desenho da Figura Humana (51,7\%, $n=15$ estudantes e 48,3\%, $n=14$ profissionais) e em terceiro o Teste Wartegg $(65,4 \%, n=17$ estudantes e $34,6 \%, n=9$ profissionais). Ao utilizar o teste Qui-quadrado, verificou-se que a distribuição entre as dez primeiras citações da amostra como um todo sobre os instrumentos psicológicos mais utilizados não era eqüitativa $\left[\chi^{2}(9,192)=30,40 ; p \leq 0,04\right]$.

O Bender Infantil e o Teste Wartegg apareceram como os mais citados como utilizados pelos estudantes e o Bender Infantil também foi o mais citado como usado pelos profissionais. Salienta-se que na ocasião da coleta esses instrumentos ainda estavam disponíveis para a comercialização, mostrando o quadro atual que ambos os instrumentos ainda não estão aprovados pelo CFP. É sabido, contudo, que o Bender Infantil encontra-se em processo de avaliação, o que pode resultar na

Tabela 3. Distribuição das freqüências e porcentagens referentes aos instrumentos psicológicos mais utilizados por estudantes e psicólogos comportamentais $(n=75)$.

\begin{tabular}{lcc}
\hline Testes mais Utilizados & $F$ & $\%$ \\
\hline Bender Infantil & 35 & 46,7 \\
Desenho da Figura Humana & 29 & 38,7 \\
Teste Wartegg & 26 & 34,7 \\
Atenção Concentrada & 18 & 24,0 \\
Escala de Maturidade Mental Columbia & 17 & 22,7 \\
O Teste Gestaltico Bender para Crianças & 16 & 21,3 \\
Teste de Rorschach & 15 & 20,0 \\
Dezesseis Fatores de Personalidade & 13 & 17,3 \\
Inventário de Interesses Angelini e Thustone & 13 & 17,3 \\
Teste Zulliger & 13 & 17,3 \\
LIP Levantamento de Interesses Profissionais & 12 & 16,0 \\
Teste de Apercepção Temática TAT & 12 & 16,0 \\
Teste Psicodiagnóstico Miocinético PMK & 12 & 16,0 \\
Bateria CEPA & 11 & 14,7 \\
BPR-5 & 11 & 14,7 \\
Escala de Inteligência Wechsler Crianças 3a edição & 11 & 14,7 \\
Inventory Multiphasic Minnesota Personality (MMPI) & 11 & 14,7 \\
Teste de Apercepção Infantil CAT-A & 11 & 14,7 \\
Inventário de Habilidades Sociais & 10 & 13,3 \\
INV & 8 & 10,7 \\
Supl. Teste Apercepção Infantil CAT-S & 8 & 10,7 \\
\hline
\end{tabular}


publicação de um parecer favorável para a utilização do teste em 2006

Watkins, Campbell e McGregor (1990), Oliveira, Noronha, Beraldo e Santarem (2003) e Oliveira et al. (2005) observam que diversos psicólogos não apresentam uma coerência teórica na escolha do instrumento, visto que optam por utilizar testes cuja fundamentação difere da abordagem adotada por esses profissionais. Assim, os resultados evidenciaram que não houve coerência entre a abordagem teórica dos estudantes e dos profissionais e a utilização de instrumentos psicológicos, tendo em vista que surpreendentemente os três testes mais utilizados pelos estudantes e psicólogos comportamentais, nesta pesquisa, possuem fundamentação na teoria tradicional da personalidade (Bender Infantil, Desenho da Figura Humana e Teste Wartegg).

Meyer (2001) destaca que se pode utilizar testes psicológicos para compor o diagnóstico comportamental. Contudo não se deve esquecer o que professa Keepe et al. (1980): os testes projetivos não devem ser utilizados na composição diagnóstica do psicólogo comportamental, visto que possuem fundamentação filosófica, metodológica e teórica divergentes da que preconiza uma avaliação comportamental.

Talvez essa falta de coerência entre a abordagem adotada e a escolha do instrumento esteja relacionada ao ensino dos instrumentos nas universidades e à formação falha nessa área. Há que ensinar aos estudantes as propriedades psicométricas do teste e sua fundamentação (Oakland, 1999) e não apenas sua aplicação e correção. Outro aspecto a ser destacado é que uma parte dos profissionais atuava em consultórios particulares e também em universidades, contudo não se havia graduado no mestrado ou doutorado na área, o que certamente poderia fazer a diferença na aprendizagem do estudante.

\section{Considerações Finais}

Este estudo teve como objetivo verificar se há diferença entre os instrumentos psicológicos mais conhecidos e utilizados por estudantes e profissionais da clínica comportamental. Observa-se que nos últimos anos a Psicologia no Brasil tem se focado nos estudos relacionados aos instrumentos psicológicos; embora não tenha sido objetivo abordar a questão da validade dos instrumentos pesquisados, parece estar claro que muitas das discussões recentes em Psicologia passam por esse âmbito. Nesse sentido, levantar as possíveis diferenças entre os instrumentos conhecidos e utilizados por estudantes e profissionais já é um passo para estabelecer se os instrumentos que aparecem com mais citações de utilização encontram-se com parecer favorável do CFP. As diretrizes elaboradas pelo Conselho Federal de Psicologia (2005) em relação aos atributos que um teste bem construído deve apresentar para que a avaliação seja válida estão sendo divulgadas nos mais diferentes âmbitos profissionais.

Este trabalho pretendeu discutir a falta de compreensão por parte de estudantes e profissionais, muitas vezes docentes, em relação aos instrumentos de medida e à abordagem adotada, no caso, a cognitivo-comportamental. Tal falta de compreensão pode ser evidenciada na alta freqüência de uso de instrumentos projetivos entre os estudantes e psicólogos comportamentais. Tal fato remete a duas considerações importantes relacionadas especialmente aos instrumentos e à formação profissional.

No que diz respeito aos instrumentos, a avaliação psicológica brasileira encontra-se em um momento especial provocado pelas resoluções recentemente promulgadas pelo Conselho Federal de Psicologia. A medida tende a proporcionar aos psicólogos, em um futuro próximo, uma possibilidade de avaliação mais eficaz ao se considerar que os instrumentos utilizados terão os requisitos mínimos exigidos quando da construção de materiais científicos, embora inicialmente possa ter provocado algum tipo de polêmica. Os últimos dois anos têm sido altamente produtivos, uma vez que pesquisadores e editores se viram obrigados a adotar os critérios exigidos pelas resoluções, gerando um maior número de pesquisas na área.

Em contrapartida, esse avanço não auxiliará nenhuma área particular da Psicologia, como a Psicologia Comportamental, ou a Psicanalítica, dentre outras. Isso significa que a aparente incoerência entre a leitura de abordagem adotada e o uso de instrumentos não se resolverá com a melhora das características técnicas dos recursos de avaliação, mas sim com uma 
conduta pessoal de cada um em relação a sua abordagem.

Para que ocorra essa minimização da incoerência, há que se melhorar a formação profissional. Essa questão não parece ser novidade nos meios acadêmicos e científicos. Eventos específicos têm sido realizados com o intuito de promover reflexões e avanços na preparação do psicólogo brasileiro. Há críticas contundentes em relação aos cursos, às ementas, aos programas, à metodologia de ensino ou ainda aos conteúdos ministrados nas disciplinas de avaliação psicológica das universidades brasileiras. É fato que ainda se prioriza as informações sobre a aplicação, a avaliação e a interpretação de instrumentos em detrimento da análise crítica ou de elementos pertinentes à construção e aos parâmetros psicométricos.

O resultado desse processo equivocado, como os dados que encontramos neste estudo, é a formação de profissionais com conhecimento bastante restrito, que dominam apenas a aplicação e a correção de poucos instrumentos. Alguns outros fatores podem ser aventados, como falta de disponibilidade de instrumentos adequados, falta de produção de conhecimentos relativos à avaliação psicológica comportamental, dentre outros tantos fatores que atingem não somente o psicólogo comportamental como a categoria profissional como um todo. O psicólogo comportamental não é exceção diante desse quadro, já que também vem comungando dessa formação e compartilhando das dificuldades presentes na área.

\section{Referências}

Alchieri, J. C., \& Bandeira, D. R. (2002). Ensino da avaliação psicológica no Brasil. In R. Primi (Org.), Temas em avaliação psicológica (pp.35-39). Campinas: Impressão Digital do Brasil Gráfica e Editora.

Alves, I. C. B., Alchieri, J. C., \& Marques, K. (2001). Panorama geral do ensino das técnicas do exame psicológico no Brasil. In Universidade Presbiteriana Mackenzie (Org.), Anais do I Congresso de Psicologia Clínica (pp.10-11). São Paulo.

Andriola, W. B. (1995). Os testes psicológicos no Brasil: problemas, pesquisas e perspectivas para o futuro. In L. S. Almeida \& I. S. Ribeiro (Orgs.), Avaliação psicológica: formas e contextos (Vol. 3, pp.77-82). Braga: APPORT.

Conselho Federal de Psicologia. (2001). Resolução no 25/2001. Acessado em dezembro 4, 2001, disponível em http://www.pol.org.br
Conselho Federal de Psicologia. (2003). Resolução no 02/2003. Acessado em março 3, 2003, disponível em http://www. pol.org.br

Conselho Regional de Psicologia - 6a Região. (2004). I Encontro de Avaliação Psicológica na Formação dos Psicólogos. Acessado em abril 15, 2004, disponível em http://www.pol.org/agenda

Conselho Federal de Psicologia. (2005). Relação dos testes psicológicos avaliados publicada pelo CFP. Acessado em julho 4, 2005, disponível em http://www.pol.org.br

Delitti, M. (2001). Análise funcional: o comportamento do cliente como foco da análise funcional. In M. Delitti (Org.), Sobre comportamento e cognição: a prática da análise do comportamento e da terapia cognitivo-comportamental (2a ed., pp.37-44). Santo André: ARBytes.

Guzzo, R. S. L. (2001). Laudo psicológico: a expressão da competência profissional. In L. Pasquali (Org.), Técnicas do exame psicológico - TEP. (Vol. 1, p.155-170). São Paulo: Casa do Psicólogo.

Haynes, S. H., \& O' Brien, W. H. (2000). Background, Characteristics and History. In S. N. Haynes \& W. H. O'Brien (Orgs.), Principles and practice of behavioral assessment (pp.3-24). New York: Kluwer Academic.

Jacquemin, J. (1995). Ensino e pesquisa sobre testes psicológicos. Boletim de Psicologia, 45 (102), 19-21.

Keepe, F. J., Kopel, S. A., \& Gordon, S. B. (1980). Manual prático de avaliação comportamental. São Paulo: Editora Manole.

Kroeff, P. (1988). Síntese de posicionamentos a serem feitos quanto ao uso de testes psicológicos em avaliação psicológica. In Sociedade de Psicologia de Ribeirão Preto (Org.). Anais da $18^{a}$ Reunião Anual de Psicologia (pp.535-537). Ribeirão Preto.

Matos, M. A. (1999). Análise funcional do comportamento. Estudos de Psicologia (Natal), 6 (3), 8-18.

Meyer, S. B. (2001). O conceito de análise funcional. In M. Delitti (Org.), Sobre comportamento e cognição: a prática da análise do comportamento e da terapia cognitivo-comportamental (2a. ed., pp.29-34). Santo André: ESETec.

Noronha, A. P. P. (2003). Estudos de validade e de precisão em testes de inteligência. Educação Paidéia, 13 (25), 25-33.

Oakland, T. (1999). Developing standardized tests. In S. M. Wechsler \& R. S. L. Guzzo (Orgs.), Avaliação psicológica: perspectiva Internacional (pp.101-118). São Paulo: Casa do Psicólogo.

Oliveira, K. L., Noronha, A. P. P., Beraldo, F. N. M., \& Santarem, E. M. (2003). Utilização de técnicas e instrumentos psicológicos: uma pesquisa com estagiários de clínica comportamental. Revista Psico - Revista da Faculdade de Psicologia da PUC-RS, 34 (1), 123-140.

Oliveira, K. L., Noronha, A. P. P., Dantas, M. A., \& Santarem, E. M. (2005). O psicólogo comportamental e a utilização de técnicas e instrumentos psicológicos. Psicologia em Estudo, 10 (1), 127-135.

Pasquali, L. (Org.) (1999). Instrumentos psicológicos: manual prático de elaboração. Brasília: LabPAM. 
Pasquali, L. (Org.) (2001). Técnicas do exame psicológico. Manual:fundamentos das técnicas psicológicas (Vol. I). São Paulo: Casa do Psicólogo.

Piotrowski, C., \& Lubin, B. (1990). Assessment pratices of health psychologist: survey of APA Division $38^{\mathrm{a}}$ Clinicians Professional Psychology: Research and Practice, 21 (2), 99-106.

Rangé, B., \& Guilhard, H. (1998). História da psicoterapia comportamental e cognitiva no Brasil. In B. Rangé (Org.) Psicoterapia comportamentale cognitiva: pesquisa, prática aplicações e problemas (pp.55-69). Campinas: Editorial Psy.

Santarem, E. M. M. (2001). Avaliação funcional do comportamento. In F. F. Sisto, E. T. B. Sbardelini \& R. Primi (Orgs.), Contextos equestões da avaliação psicológica (pp.203-218). São Paulo: Casa do Psicólogo.

Simões, M.R.S. (1995). Política e moral da avaliação psicológica: considerações em torno de problemas éticos e deontológicos. In L. S Almeida \& I. S Ribeiro (Orgs.),
Avaliação psicológica: formas e contextos (Vol. 3, pp.77-82). Braga: APPORT.

Torós, D. (1997). O que é diagnóstico comportamental. In M. Delitti (Org), Sobre comportamento e cognição: a prática da análise do comportamento e da terapia cognitivo-comportamental (pp.98-103.). Santo André: ARBytes.

Watkins, C. E. Jr., Campbell, V. L., \& McGregor, P. (1990). What types of psychological tests do Behavioral (and other) counseling psychologists use? The Behavior Therapist, 13, 115-117.

Wechsler, S. M., \& Guzzo, R. S. L. (Orgs.) (1999). Avaliação psicológica: perspectiva internacional. São Paulo: Casa do Psicólogo.

Recebido em: 27/9/2005

Versão final reapresentada em: 17/5/2006

Aprovado em: 5/6/2006 
